\title{
Medios de comunicación en internet móvil: la televisión como modelo aún pendiente de éxito
}

\author{
Por Claudio Feijóo, José-Luis Gómez-Barroso y Sergio Ramos-Villaverde
}

\begin{abstract}
Resumen: La televisión en el móvil no ha acabado de arrancar comercialmente en la mayor parte de los países europeos. No existe una única razón que explique esta situación. Antes bien, cabe referirse a un conjunto de causas complejas (e interrelacionadas). En el lado de la oferta, deben analizarse aspectos técnicos (infraestructuras, estándares), económicos (modelos de negocio, disponibilidad de contenido) y normativos. En el lado de la demanda, es necesario investigar cuál es la utilidad que el servicio realmente (y no teóricamente) ofrece al consumidor y las circunstancias en que lo usaría. El estudio de todos estos factores es el objetivo de este artículo. Se concluye que la televisión móvil asentará su presencia en el mercado sólo si se sortean algunos de los obstáculos descritos, se pone el foco en el posible usuario (y no en el producto) y se cuenta con cierto apoyo institucional.
\end{abstract}

Palabras clave: Televisión móvil, Televisión, Comunicaciones móviles, Demanda, Estándares, Modelos de negocio, Regulación, Móvil 2.0, internet móvil.

Title: Media in mobile internet: The still unsuccessful case of television

Abstract: Market response to

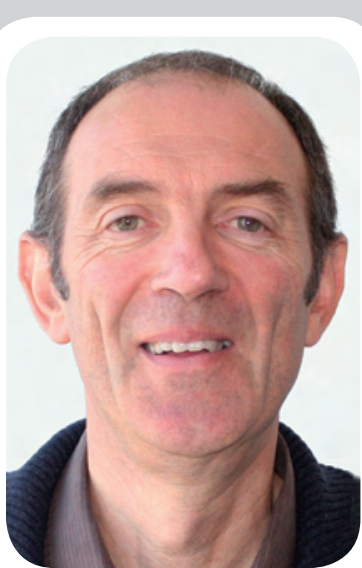

Claudio Feijóo es doctor ingeniero de telecomunicación por la Univ. Politécnica de Madrid donde es profesor titular y se dedica a investigar el impacto socio-económico de las tecnologías emergentes de la sociedad de la información, en particular aquellas relacionadas con las comunicaciones móviles y la industria de los contenidos. Entre su experiencia laboral ha disfrutado trabajando en el Institute for Prospective Technological Studies (IPTS) de la Comisión Europea y dirigiendo la Cátedra COIT sobre regulación de telecomunicaciones y políticas públicas de sociedad de la información en la UPM. Asimismo ha trabajado en muchos proyectos de investigación, desarrollo e innovación en España, Europa, Latinoamérica y norte de África, imparte conferencias y cursos de postgrado habitualmente, y es autor de numerosas contribuciones, artículos y libros sobre diversos aspectos técnicos y económicos de la sociedad de la información.

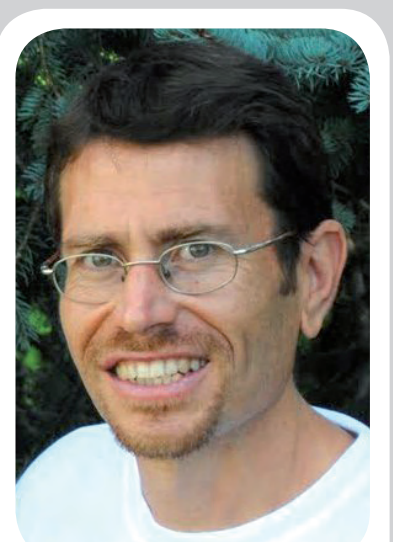

José-Luis Gómez-Barroso, profesor del Departamento de Economía Aplicada e Historia Económica de la Universidad Nacional de Educación a Distancia (UNED), es doctor y licenciado en ciencias económicas y empresariales por la UNED, ingeniero de telecomunicación por la Universidad Politécnica de Madrid (UPM) y licenciado en derecho por la Universidad Complutense de Madrid (UCM). Trabaja en dos líneas de investigación: la economía y regulación del sector convergente de las tecnologías de la información y de la comunicación, y los factores que condicionan el desarrollo de la sociedad de la información. Ha sido investigador invitado por la Universidad de Columbia, Nueva York; por la Universidad de Florida, Gainesville; por la École Nationale Supérieure des Télécommunications, París; por la Universidad La Sapienza Roma; por el Institut National des Télécommunications, Évry, Francia, y por la Fondazione Ugo Bordoni, Roma.

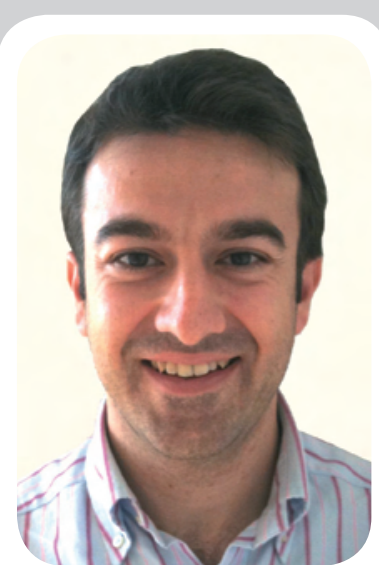

Sergio Ramos-Villaverde es ingeniero de telecomunicación y doctor ingeniero por la Universidad Politécnica de Madrid (UPM). Comenzó su carrera profesional en consultoría dentro del sector de comunicaciones electrónicas, especializándose en regulación, políticas públicas y modelos de negocio en comunicaciones móviles e internet móvil. Al mismo tiempo compaginó esta etapa con su labor docente e investigadora en la UPM, de la que fue profesor titular. En 2007 funda Noon Venture, empresa de consultoría estratégica especializada en el sector TIC, y trabaja desde diferentes puestos en las principales patronales de los operadores de telecomunicaciones en España (Aetic, Redtel) en el área de relaciones institucionales. Actualmente es director general de Noon Venture. Es Master of Business Administration (MBA) por la Stockholm School of Economics y Advanced Management Program (AMP) por el Instituto de Empresa de Madrid.

mobile television usage is quite modest in most European countries. No single reason explains this situation. On the contrary, a set of complex and interrelated causes can be found. On the supply side, the potential hurdles are in the technical (infrastructures, standards), economic (revenue models, content availability) and normative domains. On the demand side, companies must understand the (real) utility derived by consumers and the circumstances in which they intend to use the service. The conditions for mobile television success are, therefore, the research question of this paper. It is concluded that removal of these barriers, focus on the user (rather than on the product), and stronger institutional commitment are key factors for mobile television development.

Keywords: Mobile television, Television, Mobile communication, Demand, Standards, Business models, Revenue models, Regulation, Mobile 2.0, Mobile internet. 
Feijóo, Claudio; Gómez-Barroso, José-Luis; Ramos-Villaverde, Sergio. "Medios de comunicación en internet móvil: la televisión como modelo aún pendiente de éxito". El profesional de la información, 2010, noviembre-diciembre, v. 19, n. 6, pp. 637-644.

DOI: 10.3145/epi.2010.nov.10

\section{Introducción}

Recibir programas de televisión en el móvil ${ }^{1}$ es quizá el ejemplo más prominente de la tantas veces anunciada convergencia entre el subsector audiovisual de los medios de comunicación y el de los servicios de telecomunicación. Que esta convergencia progresa es indudable. Que no lo hace a la velocidad que se anunciaba, también. De hecho, pese a las expectativas que se habían alimentado en el pasado en algunos foros ${ }^{2}$ y en diversos informes ${ }^{3}$, la televisión en el móvil ${ }^{4}$ aún no ha acabado de arrancar comercialmente en la mayor parte de los países europeos e, incluso, en aquellos países en los que ha tenido un cierto éxito (Japón y Corea) se encuentra en un notorio "impasse".

No existe una única razón que explique esta situación. Antes bien, cabe referirse a un conjunto de causas complejas (e interrelacionadas) y clasificables en diversos ámbitos: técnicas, económicas, sociales e incluso institucionales. Algunos de estos obstáculos están ligados al propio servicio. Otros son "heredados" pues no debe olvidarse que no es este un mercado que se haya creado de la nada sino que se apoya en otros existentes (producción y distribución audiovisual; fabricantes de aparatos terminales; empresas operadoras de servicios de telecomunicación; creciente experiencia de los usuarios en medios interactivos) que, de un modo u otro, condicionan el desarrollo del servicio.

Ante las perspectivas que de nuevo parecen abrirse, gracias principalmente a la creciente disponibilidad de banda ancha móvil y de terminales atractivos para el uso de información audiovisual (Feijóo, Gómez-Barroso, 2009), parece necesario examinar cuál es la solidez de las barreras que han impedido el desarrollo del mercado en el pasado y qué nuevos factores han de ser tenidos en cuenta. Es el objetivo que se plantea en este artículo.

Se comienza aclarando cómo puede llegar la señal de televisión al móvil. No es una mera cuestión técnica: la evolución del mercado no es independiente de las opciones tecnológicas que existen. A continuación, se entra en la descripción de las causas que han frenado (y frenan) la difusión del servicio. De un lado se han agrupado las referidas al lado de la oferta, es decir, las que afectan a la puesta a disposición del servicio ante los consumidores, y, del otro, las vinculadas con la demanda, a saber, todas las que pueden dificultar que los consumidores lo acepten. En realidad se trata de una división a efectos didácticos pues la oferta se modifica en función de la respuesta de la demanda que, a su vez, se transforma con los cambios en la oferta. La discusión sobre la trascendencia y vigencia de estas barreras, y una conjetura sobre cuál pueda ser el futuro próximo de la televisión en el móvil, cierran el artículo.

\section{Alternativas técnicas para la televisión móvil y situación actual}

Un programa de televisión puede llegar al móvil por dos vías diferentes: mediante radiodifusión (pero de señales de televisión "adaptadas" para ser recibidas en dispositivos móviles) o a través de una conexión de banda ancha ofrecida por una red de comunicaciones móviles celular. La distinción no acaba en el campo de lo técnico: las cadenas de valor asociadas son radicalmente diferentes.

En el primero de los casos no hay diferencias conceptuales con la televisión convencional salvo la adecuación de la señal enviada que exige el aparato receptor. Como ocurre en la televisión, los ingresos se basarían en la publicidad o, en el caso de canales codificados, en una cuota de suscripción o en pago por programa. La cadena de valor mantiene, en esencia, paralelismo con la actual (figura 1).

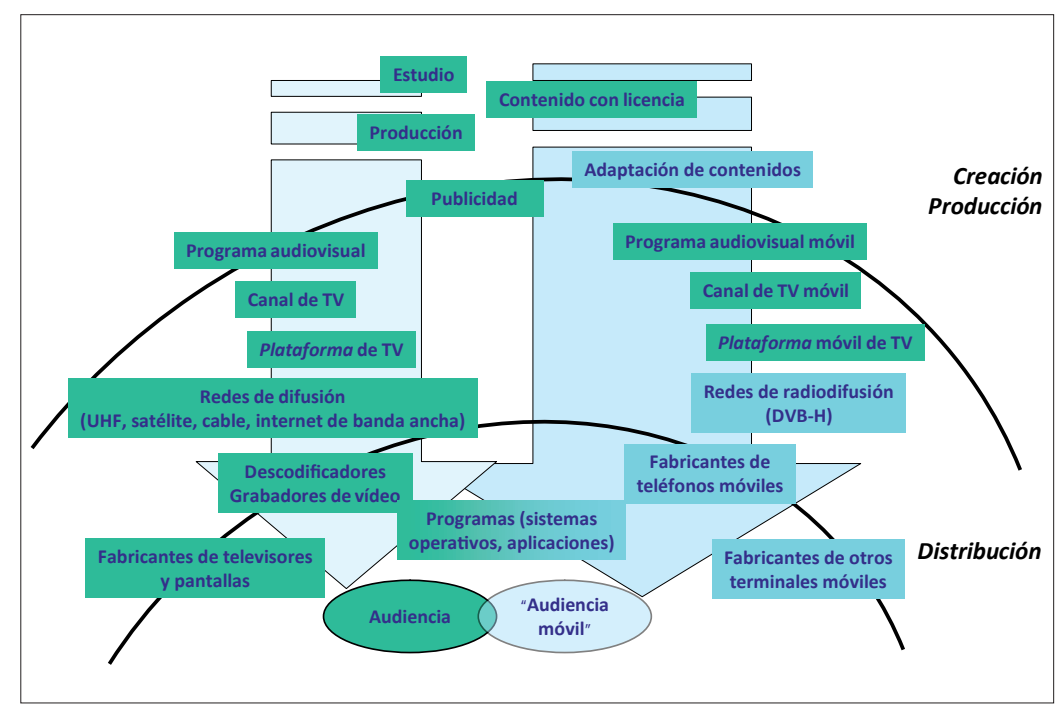

Figura 1. Cadena de valor de la radiodifusión: televisión convencional y para móvil 
Si se utiliza la red de una compañía de comunicaciones móviles hay tres maneras posibles de enviar la señal. La más utilizada actualmente, con diferencia, es la transmisión personalizada (se suele conocer por el vocablo inglés unicast) en que se envía a cada usuario el programa que solicita ${ }^{5}$. Puesto que la señal de vídeo (de televisión) suele ocupar una parte importante del ancho de banda que ofrecen las comunicaciones inalámbricas (hoy por hoy bastante más limitado que el de las redes fijas), podrían existir problemas de congestión cuando coinciden muchas solicitudes. Una solución es la difusión simultánea (multicast) en que se emite el mismo programa a un grupo de usuarios que pierden, como contrapartida, algunos grados de libertad para elegir una "televisión a la carta". La tercera vía usa técnicas de intercambio entre clientes (peer to peer) pero es una área aún necesitada de desarrollo técnico.

Desde el punto de vista del negocio existen dos industrias separadas (y dos intereses) que confluyen en una capa próxima al consumidor del servicio final (figura 2). Las posibilidades para la obtención de ingresos incluyen, también aquí, la publicidad, la suscripción y el pago por visión pero se pueden plantear, además, diversos tipos de empaquetamiento con servicios de voz y de datos, al estilo del denominado "triple play" de la banda ancha fija.

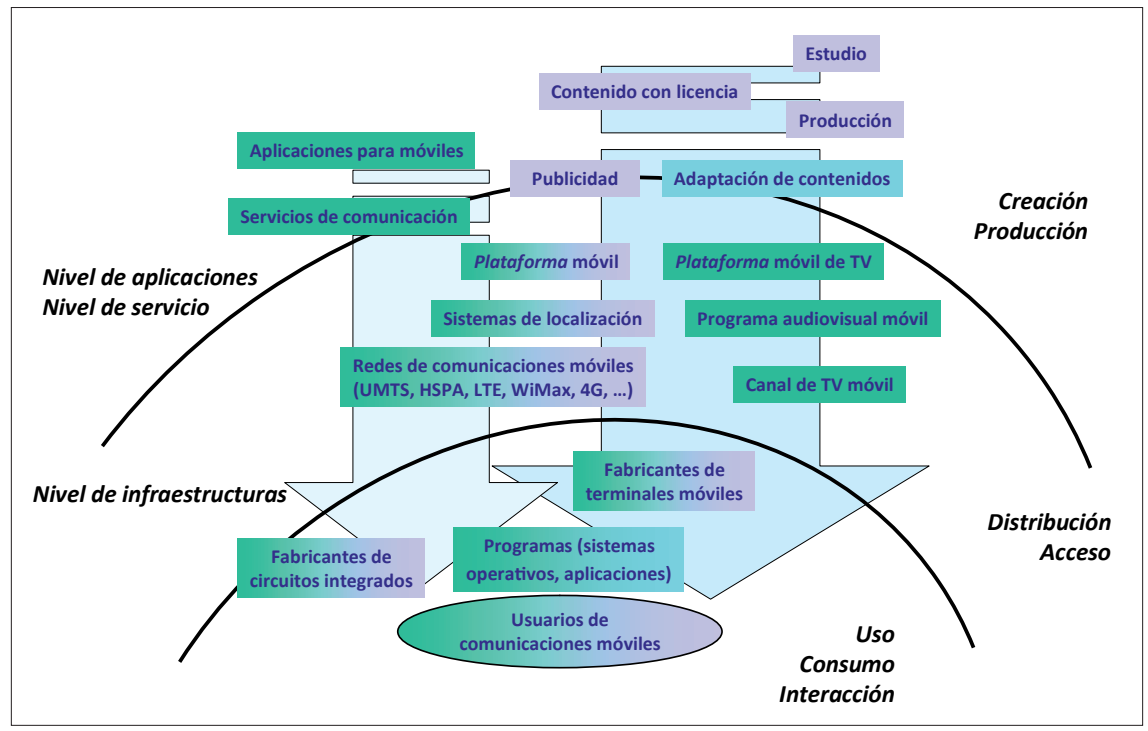

Figura 2. Cadena de valor de comunicaciones móviles: servicios genéricos y televisión centran en la información de vídeo. Por su lado, los operadores de comunicaciones móviles pretenden imponer sus condiciones frente a los agentes del sector audiovisual, haciendo de la televisión un atractivo más de sus accesos de banda ancha. Finalmente, los fabricantes de dispositivos móviles quieren que las opciones de acceso para los usuarios sean las más variadas posibles, pero que las aplicaciones de valor añadido que se desarrollen a partir de información audiovisual lo hagan sobre sus plataformas.

Las relaciones entre todos estos agentes son complejas. Que las compañías de comunicaciones móviles necesitan a los productores audiovisuales está fuera de duda. Igual sucede con los fabricantes. Pero la dependencia también se da en el otro sentido: incluso el primero de los modelos no deja fuera a las empresas de móviles. En las preguntas frecuentes que se contestan en el Fórum $D V B-H$ (véase el significado de la sigla más adelante) se dice que "hay muchas razones por las que una colaboración [con los operadores móviles] sería juiciosa. Por ejemplo, en muchos países los teléfonos están subsidiados por los operadores. También la facturación, elemento clave, para la que los operadores tienen sistemas ya aceptados en el mercado".

Las últimas cifras de usuarios disponibles recopiladas por los autores a partir de diversas fuentes del sector y referidas a 2009, aparte de ser mucho más modestas que las previsiones que regularmente se efectúan, hablan de una notoria segmentación por regiones con respecto a las diversas alternativas tecnológicas. Así, en Europa existen unos 5 millones de usuarios casi todos ellos basados en los sistemas de comunicaciones móviles, una situación relativamente parecida a los EUA donde se alcanzan los 10 millones de usuarios. Por el contrario en Japón y Corea hay unos 60 y 30 millones de usuarios respectivamente con una clara dominancia de los sistemas de radiodifusión, aunque son las ofertas basadas en comunicaciones móviles las que están creciendo más rápidamente en

Del examen de cada uno de estos esquemas resulta obvio que cada empresa interesada en el mercado de la televisión móvil lucha por que el valor (y por tanto el ingreso asociado) se desplace hacia la parte sobre la que mantienen el control. Las cadenas de televisión asentadas preferirían, por tanto, el primer modelo en que se otorga a la red un mero papel instrumental. El mismo objetivo tienen los portales de internet que se la actualidad. En España según los últimos datos disponibles para el año 2009 (CMT, 2010) existían 350.000 abonados a la televisión de pago móvil (300.000 en el año 2007) que generaron ingresos para los operadores por valor de 19 millones de euros (9 millones en 2007), lo que significaba el $0,5 \%$ de los ingresos totales de la televisión en España. Movistar tenía el 68\% de la cuota de mercado, Vodafone el $28 \%$ y Orange el $4 \%$. 


\section{Barreras en el lado de la oferta}

\subsection{Aspectos técnicos: infraestructuras y estánda-} res

El requisito primero es el despliegue de infraestructuras capaces de transmitir la señal. En Europa, en general, la radiodifusión de televisión móvil no ha ido más allá de experiencias puntuales. En cambio, las redes inalámbricas de banda ancha están extendiéndose y aumentando su capacidad con ambiciosos planes para el desarrollo de la llamada cuarta generación de comunicaciones móviles ya en marcha (GSA, 2010).

Cómo transmitir esa señal es la segunda parte de la historia. En la mayor parte de los mercados tecnológicos (servicios, productos, aplicaciones), el impulso que sigue a la fase de introducción requiere la existencia de estándares ampliamente aceptados que permitan a los fabricantes o diseñadores aprovechar las economías de escala. Tales estándares no existen en la televisión móvil, mercado aún inmaduro en que diferentes normas pugnan por imponerse al resto.

Así, para el caso de la radiodifusión, la Comisión Europea se adhirió al estándar $D V B-H$ en noviembre de 2007, lo que se suponía que lo convertiría de facto el estándar europeo, algo que no ha llegado a suceder y que de hecho se encuentra más bien en un "limbo" (Grivet, 2009). Pero además existen muchas otras normas, como $D V B-S H$ que combina las posibilidades de difusión terrestre y por satélite, la coreana $D M B$, la japonesa ISDB-T, MediaFlo popular en EUA y la China $S T i M i^{6}$. Otras regiones (Iberoamérica por ejemplo) no han optado por una norma común. Para complicar más el escenario, ya en 2008 se anunció que los dispositivos móviles serán en el futuro capaces de "entender" $D V B$ $T$, el estándar de la TDT.

En la segunda opción, los operadores móviles están en plena fase de extensión, pero también de renovación o transformación, de sus redes. El problema de los estándares está presente en partes medulares de estas redes y, lo que es más importante en este ámbito, existen incertidumbres acerca de cuál debe ser el ritmo de despliegue (cómo recuperar la inversión) y de cuál sea el impacto producido por el uso intensivo de aplicaciones basadas en vídeo en la capacidad de estas redes ${ }^{7}$. De hecho este último es el mayor y mejor argumento para las redes de difusión de televisión móvil. Según Grivet (2009) el coste por usuario en una red de difusión será alrededor de diez veces menor que una red de comunicaciones móviles con "unicast".

\subsection{Aspectos económicos: modelos de negocio}

La elección del modelo de negocio más apropiado ha gravitado entre suscripción, pago por visión (o por descarga) y publicidad a lo largo de la corta historia de la televisión móvil. Y aunque la cuestión dista de estar clara, algunos datos recientes arrojan alguna luz sobre el panorama.

Utilizando datos de hasta once informes diferentes sobre el estado de la televisión móvil se han hecho las estimaciones que se presentan en la figura 3 (donde se han incluido también la música y los juegos en el móvil por comparación). Aquí, al igual que en otros ámbitos móviles emergentes (Feijóo, Gómez-Barroso, Martínez, 2010), aparece la publicidad como ganadora neta. Se dan unos ingresos para la televisión en el móvil de alrededor de 10.000 millones de dólares en 2013, de los cuales aproximadamente tres cuartas partes lo serían vía publicidad, con un crecimiento anual medio que se situaría entre el 20 y el $25 \%$ según las mismas fuentes. Este dato parece quedar corroborado por el sonado fracaso del modelo de suscripción sobre redes específicas de difusión de televisión móvil: a finales de 2009 existían tan solo 3,2 millones de usuarios suscritos a esta modalidad en todo el mundo (Holden, 2010). De hecho el modelo de difusión de televisión móvil más exitoso es el japonés que está basado en la transmisión "abierta" de televisión.

También la publicidad aparece como la ganadora en las formas de vídeo en el móvil no tradicionales, donde compañías como YouTube están intentando transferir sus incipientes modelos de negocio de internet al ámbito móvil, utilizando como base la fuerza creadora de los propios usuarios móviles (Feijóo, Pascu, Misuraca, Lusoli, 2009). Pero como la propia figura 3 indica éste es todavía un área menor desde el punto de vista del negocio.

El único otro modelo de negocio que presenta alguna novedad es el de las aplicaciones móviles típicamen-

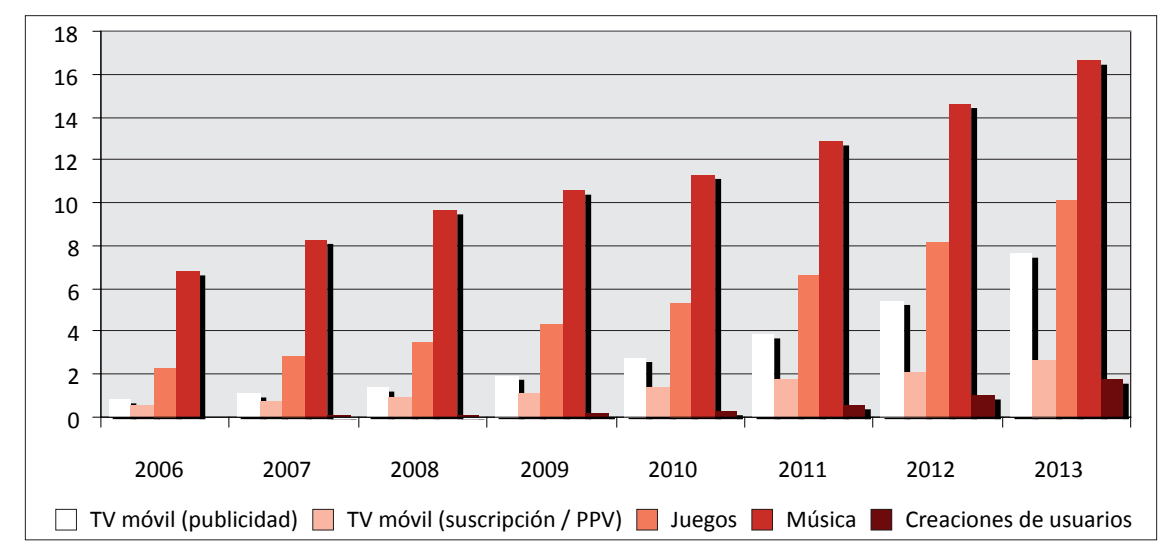

Figura 3. Ingresos reales (2006 - 2008) y esperados para diferentes servicios móviles en todo el mundo (millardos de dólares). Basado en datos de empresas de consultoría especializada en el ámbito móvil (ABI Research, eMarketer, Informa Telecoms and Media, Juniper Research, Netsize, PWC y Research and Markets) 
te adquiridas por los usuarios a través de algunas de las tiendas existentes (AppStore de Apple, Android Market de Google, Ovi de Nokia, etc.), un segmento que alcanzaría los 14 millardos de dólares en 2013 (Idate, 2010). En el caso de la televisión móvil, en general se trata de aplicaciones que hacen meramente de vehículo para acceder a los contenidos audiovisuales, pero es cierto que se plantea, al menos teóricamente, la posibilidad de ofrecer elementos de valor añadido dentro de la propia aplicación al estilo de la venta de canciones dentro de un juego móvil por poner un ejemplo conocido. Otra vez la utilización de este modelo dentro del ámbito de la televisión (o incluso el vídeo) móvil, está por suceder.

El ámbito de los modelos de negocio se completa con la cuestión de la disponibilidad de contenidos. Aquí, los conflictos referidos a la obtención de derechos o a la forma de protegerlos son problemas que se agudizan en el caso de la televisión móvil (Screen Digest, CMS Hasche Sigle, Goldmedia, \& Rightscom, 2006). No es obvio cómo conciliar los intereses de unas compañías necesitadas de ofrecer, especialmente en las primeras etapas de desarrollo del mercado, unos contenidos atractivos a precios muy asequibles con los de unos distribuidores que solicitan compensaciones adicionales por ceder sus materiales para que sean explotados en un nuevo canal.

\subsection{Aspectos normativos: doble regulación}

En el servicio de la televisión móvil confluyen dos ordenamientos complejos: el que se aplica a la televisión (audiovisual) y el de los móviles (telecomunicaciones). $\mathrm{Y}$ es que la convergencia normativa entre los dos mundos, aunque muchas veces anunciada, está aún lejos de producirse.

Puesto que se trata de un medio audiovisual, se ha de considerar cómo se trasvasan a este nuevo canal las condiciones impuestas a los restantes: la protección de valores fundamentales, la vigilancia del pluralismo de los medios de comunicación, los límites al uso de la publicidad o la promoción de la diversidad cultural son factores que sin duda afectarán al éxito del servicio (Bria, Kärrberg, Andersson, 2007). Más aún, ¿es necesario extender el servicio público al mundo móvil? ${ }^{8}$.

Puesto que se trata de un servicio de telecomunicación, se han de fijar las condiciones bajo las que los diferentes agentes pueden entrar y operar en estos mercados y se ha de vigilar cuándo su actividad es sospechosa de entorpecer el desarrollo del mercado. También aquí el papel de los reguladores va más allá del de mero árbitro que establece unas reglas de juego básicas $\mathrm{y}$, conscientes de ello o no, pueden ayudar a "orientar" hacia un determinado resultado, Este es el caso del "dividendo digital" que resulta del proceso de digitalización de la señal de televisión difundida por ondas terrestres. Prácticamente todos los servicios y aplicaciones que hacen uso del espectro radioeléctrico estén interesados en participar en su reparto. Los canales de televisión hacen presión para que se siga manteniendo su uso primitivo y se utilice para aumentar el número de canales de TDT. Los operadores de comunicaciones móviles han entrado en la pugna. E incluso no sería completamente descartable que una empresa nueva, interesada en ofrecer televisión en el móvil, pujara en las circunstancias apropiadas por una parte del dividendo si éste, como en algunos otros países europeos, se saca a subasta. Lo que es claro es que la televisión móvil necesita espectro (Ramos, Pérez, Mascarell, 2007) y ésta parece ser la mejor oportunidad próxima para que lo consiga.

\section{Barreras en el lado de la demanda: percepción de los usuarios}

Es regla para todo bien o servicio que se lanza al mercado que no basta con ponerlo al alcance de los consumidores. Es necesario además, claro está, ganar su favor. Al menos, el favor de un número de consumidores tal que la rentabilidad justifique la inversión. Cuando esto no ocurre, la causa suele ser simple: el producto no resulta atractivo.

No parece este el caso de la televisión móvil, al menos en ciertos países como los nórdicos. En la encuesta de Westlund (2008), realizada en Suecia, el número de usuarios potencialmente interesados alcanza el 33\%; Verkasalo (2008) da un valor del $16 \%$ para Finlandia. Pero lo cierto es que pocos están usando el servicio en esos países. Los mismos trabajos dan un 8\% de usuarios en Finlandia y sólo un 3\% en Suecia. Puede pensarse en el precio como motivo que explique este desajuste entre interés teórico y adopción real. Sin embargo, el precio no es la razón principal (o al menos no la única). Cabe aquí citar una experiencia llevada a cabo en el Reino Unido, en donde la $B B C$ lanzó una emisión en pruebas (gratuita) de mayo de 2007 a abril de 2008: el desalentador resultado fue una audiencia diaria máxima de unas 600 personas con una media de 13 minutos ¡al mes!?.

El caso de España no es diferente en cuanto al precio. Según los últimos datos disponibles (de la encuesta Consumers and convergence que ha realizado la consultora $K P M G$ en 2010 a 5.500 usuarios de móvil de 22 países) un considerable $29 \%$ de los usuarios de internet móvil estaría dispuesto a pagar por contenidos. Aquí las diferencias culturales entre países se manifiestan claramente: en Holanda solo estarían dispuestos a pagar un $6 \%$, en Irlanda un $12 \%$ y en Alemania el $17 \%$. 
Parecería existir, entonces, un desencuentro entre oferta y demanda, como sugieren Åkesson y Eriksson (2007) (quienes hablan de "falta de engranaje" entre medios de comunicación móviles y modelos de uso), Carlsson et al. (2006) (quienes extienden el problema a los contenidos para móviles en general) o GarcíaGarcía et al. (2010) (quienes abundan en la cuestión de la interacción y no del consumo). Y es que es necesario que las empresas entiendan las peculiaridades de un servicio para el que, pese a su nombre, no sirve el conocimiento que se tiene sobre los parámetros de consumo ni de la televisión ni de las comunicaciones móviles.

Obviamente, la programación cuenta. A partir de un estudio también hecho en Finlandia, Carlsson y Walden (2007) describen el programa tipo seleccionado por los usuarios como aquel en que la información puede ser asimilada en menos de diez minutos (noticias, deportes, dibujos animados, documentales), nunca con duración superior a 45 minutos y preferiblemente almacenable en el móvil para ser visto en el momento que se elija. Precisamente respecto al momento, a la pregunta crucial de cuándo y cómo se vería la televisión móvil, Tadayoni et al. (2008) describen tres simulaciones realizadas en Europa: en Helsinki, la televisión móvil se veía durante los desplazamientos al o desde el trabajo (o la universidad) aunque la conexión en casa (y en el lugar de trabajo) era "sorprendentemente alta"; en diversos lugares de España, "en casa" fue la situación preferida al inicio del experimento aunque acabó siendo superada por los desplazamientos; en el tercero de los casos, en Oxford, se cuantificó el momento en que más tiempo se permanecía conectado, que resultó ser "durante los desplazamientos" para un $37 \%$ de los participantes, seguido de "en casa" (32\%), "en el trabajo" (23\%) y otras situaciones (8\%). En todos estos simulacros, las dos razones con más frecuencia argüidas para el uso fue "matar el tiempo" y "mantenerse informado".

Fuera de Europa, Corea del Sur es un ejemplo especialmente interesante. En este país compite la radiodifusión (tanto gratuita como de pago) con el servicio que tres compañías operadoras de servicios móviles ofrecen a sus clientes (televisión interactiva tanto en pago por visión como en suscripción mensual). ChanOlmsted et al. (2008) dan allí una media de dos a tres días de uso a la semana (unos 30 minutos al día), utilizándose primordialmente como segunda televisión en casa y también durante los desplazamientos; otro dato de interés es que el $50 \%$ de los encuestados estaba dispuesto a usar el servicio si era gratis y que un $68 \%$ no estaba dispuesto a pagar en ningún caso. De hecho, en el estudio de Choi et al. (2008), realizado también en Corea del Sur, el coste es uno de los dos atributos más importantes en que se fijan los usuarios; el otro es la calidad del contenido. En el mismo país, Jung et al.
(2009) añaden otros factores necesarios para adoptar el servicio como la facilidad de uso y la "utilidad percibida".

Una vía alternativa para comprender la actitud del usuario (o, mejor, de una parte significativa de los usuarios) ante la televisión móvil, es la abierta por Carlsson et al. (2006). Estos autores defienden que si la aparente ventaja de un servicio móvil (ubicuidad y accesibilidad en cualquier momento) no siempre se refleja en los parámetros de uso, ello es debido a que el uso del móvil está más ligado con la experiencia de contactar con gente y con el conocimiento del entorno que con el entretenimiento, y que por ello los usuarios han de encontrar un "contexto" (temporal, espacial, situación emocional) para usar un determinado servicio. De hecho, la situación "involucrarse socialmente vía el móvil a la vez que abandonar otros vínculos sociales" es una de las dos paradojas (de ocho consideradas) que Loebbecke et al. (2008) afirman que hay que tener en cuenta en el caso de la televisión móvil.

Avanzando por esta vía, se podría llegar a un trasvase del concepto de web 2.0 - móvil 2.0 para llegar a lo que Schatz et al. (2007) denominan "televisión móvil social" y que definen como "una forma de comunicación que crea una experiencia compartida de visión de televisión en el móvil" y donde los usuarios son los innovadores, aunque la forma exitosa de ponerla en marcha es aún desconocida y tropieza con importantes barreras (Nicolajsen, Sørensen, 2009). Datos recientes para el caso de España (Fundación Telefónica, 2009) coinciden en señalar que la mayor fuente de contenidos audiovisuales consumidos en el móvil son aquellos grabados por los propios usuarios (49\%) frente a porcentajes en torno al 10\% para los servicios audiovisuales de los propios operadores o de otros proveedores de servicios.

\section{Conclusión: retos para el desarrollo de la televisión en el móvil}

Durante los últimos años se han repetido los anuncios que pronosticaban un futuro brillante (y próximo) para una televisión "rejuvenecida": la televisión en el móvil. Todos estos pronósticos se fijaban en la imagen que se veía en la caja del rompecabezas, sin reparar (o sin querer reparar) que la caja estaba apenas abierta y las piezas amontonadas. Encontrarlas y juntarlas no está siendo un proceso fácil. Algunos trozos están ensamblados pero aún no es fácil adivinar cuál sea el cuadro final.

Así, en el centro del rompecabezas, son cada vez más las personas que tienen en su bolsillo un aparato válido para que ver televisión en él sea un acto frecuente y no la última opción desesperada para intentar no 
perderse la final del evento deportivo. Baterías que "no se mueren" tan pronto se reciben las primeras imágenes, pantallas nítidas y sin reflejos y manejo fácil son ingredientes en esta receta.

Lo que rodea a este centro no está del todo montado. Los diversos proyectos en pugna no están claramente definidos (estándares, estrategias, modos con que obtener rentabilidad) y causan en el usuario confusión y cierto rechazo. Hasta el usuario han estado llegando ofertas a veces complicadas y, las más de las ocasiones, caras. No se trata de que se imponga una única opción (la competencia es positiva) pero sí de que las diferentes iniciativas lleguen a una mayoría de la audiencia potencial y no presenten excesivas complicaciones (del tipo "poseer el aparato adecuado y ser cliente del operador correcto con el tipo de contrato apropiado para que el precio...").

En la actualidad, el modelo basado en la radiodifusión está aún en un estado comercial muy incipiente en Europa. La televisión móvil es, entonces, la que se ofrece por las redes de los operadores móviles. Y estas compañías, en general, han apostado tibiamente por el servicio, probablemente con la intención de ir graduándolo para así intentar mantener el control de todo lo que por sus redes se ofrece, a la par que evitar su saturación. Con el agravante de que, aunque el canal de televisión sea gratuito, el envío de datos no lo es y no ha sido hasta muy recientemente cuando han comenzado a generalizarse las tarifas planas de banda ancha (que tienen por ahora siempre limitaciones en el ámbito móvil). Tarifas planas que no existen cuando se cambia de país (al contrario, las tarifas de itinerancia son muy abultadas), coartando así una demanda que podría ser importante (casi cualquiera querría ver la televisión local cuando está fuera). A todo lo anterior hay que añadir las complicaciones resultantes de la transferencia y protección de derechos y de unas regulaciones no siempre homogéneas.

Con todo, y como ocurre en cualquier rompecabezas, lo más difícil no es juntar las piezas del castillo sino las del fondo. Y parece que muchos de las compañías que han lanzado su oferta al mercado se han preocupado del edificio pero no del lugar en que está emplazado. Que el producto es tan atractivo "que se venderá solo" es un axioma peligroso. Recuérdense ejemplos pasados, como la videoconferencia. Si no se entiende cuál es la utilidad que el servicio realmente (y no teóricamente) ofrece al consumidor (por qué puede estar interesado, en qué circunstancias lo usaría), de poco servirá el resto. Alcanzar el punto de inflexión en que la demanda de los pioneros se convierte en aceptación masiva no es fácil. Y no debe jamás confundirse la disposición animosa de los precursores con la que es la actitud de la audiencia "media".
Precisamente ahora parecería estarse cerca de ese punto. Los próximos dos o tres años van a ser clave para saber hacia dónde se camina. Si se cuenta con cierto apoyo institucional (particularmente en cuestiones regulatorias), se sortean algunos de los obstáculos descritos y se pone el foco en el posible usuario y no en el producto, la televisión móvil asentará, ahora sí, su presencia en el mercado. De lo contrario, no desaparecerá pero en muchos lugares continuará siendo cuestión de minorías.

Agradecimiento: Este artículo está basado en parte en los trabajos del proyecto de investigación Evolución del medio móvil en España (CSO2009-07108).

\section{Notas}

1. Con "el móvil" haremos referencia a todo tipo de aparatos transportables que sirven para recibir servicios basados en comunicaciones inalámbricas, en particular la televisión. La lista incluye, claro está, teléfonos móviles pero también agendas electrónicas, miniordenadores o miniconsolas.

2. Tómense, como ejemplo, las declaraciones de Rob Hyatt, director de "contenido móvil" de la compañía operadora de telecomunicaciones estadounidense Cingular, en el número de BusinessWeek del 11 de octubre de 2005: "en teléfonos móviles, la demanda de aplicaciones ligadas con la visión de vídeos podría incluso superar la de videojuegos, 'tonos' y fondos de pantalla e involucrar a prácticamente al 100\% de la población”. Declaraciones de este tono han abundado hasta fechas muy recientes.

3. Datamonitor (2007) preveía para la televisión transmitida por redes de radiodifusión 65 millones de usuarios en 2010 en todo el mundo y $155 \mathrm{mi}$ llones dos años más tarde; Informa Telecoms and Media (2008) hablaba, para esos mismos años, de 600 y 1.000 millones de usuarios para la televisión ofrecida por las redes móviles.

4. Con "televisión en el móvil" hemos preferido utilizar la expresión más frecuente para referirse al consumo general de "información audiovisual en el móvil".

5. Esto da posibilidades que no presenta la difusión general y hace que se trate más de vídeo móvil que de televisión móvil. Se puede interpretar como "televisión a la carta" o "televisión personalizada".

6. El lector interesado en la comparación entre los diferentes estándares puede consultar por ejemplo Zhou et al. (2009)

7. Según Cisco, el tráfico en las redes de los operadores móviles se va a multiplicar por 70 veces entre 2008 y 2018 (Grivet, 2009).

8. Lo que se toparía además con el sistema de financiación seguido en algunos países en que se cobra una tasa por la posesión de un televisor (Hämmäinen, 2007). ¿También por la posesión de un teléfono?

9. http://www.pocket-lint.co.uk/news/news.phtml/16463/17487/BBCMobile-TV-trial-flunks.phtml

Cabe matizar la cuestión del precio. El experimento se llevó a cabo no mediante radiodifusión sino mediante acuerdos con los operadores móviles de país con lo que los usuarios no pagaban por el servicio de televisión pero sí por el del envío de datos (en caso de no tener contratada una tarifa plana).

10. Ciertos servicios no son meros pasos adelante o "adaptaciones" a un nuevo entorno. A este respecto, Jenson (2005) critica que los MMS, otra aplicación que utiliza imágenes, fueran en su momento considerados una extensión o "progreso natural" de los SMS; el tiempo se ha encargado de demostrar que, en efecto, su uso responde a diferentes motivaciones y se rige por diferentes pautas.

\section{Bibliografía citada}

Åkesson, Maria; Eriksson, Carina I. "The vision of ubiquitous media services: how close are we?". In: Human interface and the management of information. Interacting in information environments: Berlin / Heidelberg: Springer, 2007. 
Bria, Aurelian; Kärrberg, Patrik; Andersson, Per. "TV in the mobile or TV for the mobile: challenges and changing value chains". Proc of Pimrc 2007. IEEE $18^{\text {th }}$ Intl symp on personal, indoor and mobile radio communications, 2007

Carlsson, Christer; Carlsson, Joanna; Puhakainen, Jussi; Walden, Pirkko. "Nice mobile services do not fly. Observations of mobile services and the Finnish consumers". e Conference, eValues, 2006.

Carlsson, Christer; Walden, Pirkko. "Mobile TV - To live or die by content". Proc of Hicss 2007, 40th Annual Hawaii intl conf on system sciences, 2007.

Carlsson, Christer; Walden, Pirkko; Bowman, H. "Adoption of 3G+ services in Finland". Intl journal of mobile communications, 2006, v. 4, n. 4, pp. 348-361.

CMT (2010). Informe anual 2009. Barcelona: Comisión del Mercado de las Telecomunicaciones.

http://www.cmt.es/es/publicaciones/anexos/20100705_IA09_CMT_ INFORME_ANUAL_2009_SENCER_BAIXA.pdf

Chan-Olmsted, Sylvia M.; Lee, Sangwon; Heo, Jun. "Developing a mobile television market: lessons from the world's leading mobile economy - South Korea". En: 8th World media economics and management conf., 2008.

Choi, Jae Young; Koh, Daeyoung; Lee, Jongsu. "Ex-ante simulation of mobile TV market based on consumers' preference data". Technological forecasting and social change, 2008, v. 75, n. 7, pp. 1043-1053.

Datamonitor. The mobile broadcast TV market 2007.

http://www.datamonitor.com/industries/research/?pid=BFTC1483

Feijóo, Claudio; Gómez-Barroso, José-Luis. "Factores clave en el acceso móvil a contenidos". El profesional de la información, 2009, mar.-abril, v. 18, n. 2, pp. 145-154.

Feijóo, Claudio; Gómez-Barroso, José-Luis; Martínez-Martínez, Inmaculada J. "Nuevas vías para la comunicación empresarial: publicidad en el móvil". El profesional de la información, 2010, v. 19, n. 2, pp. 140-148.

Feijóo, Claudio; Pascu, Corina; Misuraca, Gianluca; Lusoli, Wainer. "The next paradigm shift in the mobile ecosystem: mobile social computing and the increasing relevance of users". Communications \& strategies, 2009, n. 75 (3rd quarter), pp. 57-78.

Fundación Telefónica. Informe de la sociedad de la información en España. Madrid, 2009.

http://e-libros.fundacion.telefonica.com/sie09/

García-García, Alberto; Vinader-Segura, Raquel; Albuin-Vences, Natalia. "Televisión tradicional y televisión móvil. Estrategias para contenidos televisivos en movilidad". Telos, 2010, n. 83, pp. 84-96.

Grivet, Vincent. "Business models in mobile TV". In: Paris Tech Telecom, 2009 .

GSA. GSM/3G market update, 2010

http://www.gsacom.com/downloads/pdf/GSM_3G_Market_Update.php4

Hämmäinen, Heikki. "Finland counting on DVB-H". Nordic and Baltic journal of information and communications technologies, 2007, v. 1, n. 1, pp. $12-15$.

Holden, Windsor. Tuning in to mobile TV. Basingstoke, UK: Juniper Research, 2010

http://www.juniperresearch.com/shop/products/whitepaper/pdf/JRL\%20\%20MTV10\%20\%20White\%20Paper\%20-\%20sec.pdf

Idate. Digiworld yearbook. The digital world's challenges. Montpellier, 2010.

Informa Telecoms and Media. Mobile social networking: communities and content on the move, 2008.

http://isg-stage-nat.informa.com/marlin/30000001001/DOWNLOAD/mar ketingid/20001626480/fileid/20001509146? proceed $=$ true \&Mar EntityId=1231159648928\&entHash=102b2b8aela
Jenson, Scott. "Default thinking: why mobile services are setup to fail". In: R. Harper, Palen, L., Taylor, A. (Ed.), The inside text: social, cultural and design perspectives on SMS, Springer, 2005.

Jung, Yoonhyuk; Pérez-Mira, Begoña; Wiley-Patton, Sonja. "Consumer adoption of mobile TV: Examining psychological flow and media content". Computers in human behavior, 2009, v. 25, n. 1, pp. 123-129.

Loebbecke, Claudia; Huyskens, Claudio; Jarvenpaa, Sirkka L. "Adoption of mobile TV services among early users: Convergence of familiar technologies and emergence of technology induced paradoxes". In: Proc of ICMB '08. $7^{\text {th }}$ Intl Conf on Mobile Business, Barcelona, 2008.

Nicolajsen, Hanne W.; Sørensen, Lene T. "User as innovators - user needs for future converged mobile media services". In: Proc of the $32^{\text {nd }}$ Information systems research seminar in Scandinavia, 2009.

Ramos-Villaverde, Sergio; Pérez, Jorge; Mascarell, Borja. "Mobile TV services provision scenarios - implications of spectrum and audiovisual policies in the development of mobile TV market in Europe". The journal of the communications network, 2007, n. 6 (Part I Jan.-Mar. 2007), pp. 40-47.

Screen Digest, CMS Hasche Sigle, Goldmedia \& Rightscom. Interactive content and convergence: implications for the information society. London: European Commission. DG Information Society and Media (S. Digest), 2006.

Schatz, Raimund; Wagner, Siegfried; Egger, Sebastian; Jordan, Norbert. "Mobile TV becomes social - Integrating content with communications". In: Proc of ITI 2007, $29^{\text {th }}$ Intl conf on information technology interfaces, Zagreb (Croacia), 2007.

Tadayoni, Reza; Henten, Anders; Skouby, Knud E. "Mobile TV as part of IMT advanced: technology, market development, business models and regulatory challenges". Wireless personal communications, 2008, v. 45, n. 4, pp. 585-595.

Verkasalo, Hannu. "From intentions to active usage: a study on mobile services in Finland". In: 19th European regional conf of the intl telecommunications society, 2008.

Westlund, Oscar. "Diffusion of internet for mobile devices in Sweden". Nordic and Baltic journal of information and communications technologies, 2008, v. 2, n. 1 , pp. 39-47.

Zhou, Jiehan; Ou, Zhonghong; Rautiainen, Mika; Koskela, Timo; Ylianttila, Mikka. "Digital television for mobile devices". IEEE multimedia, 2009, v. 16, n. 1, Jan.-Mar., pp. 60-70.

Claudio Feijóo, Institute for Prospective Technological Studies, Joint Research Centre, European Commission.

Inca Garcilaso, 3. 41092 Sevilla. claudio.feijoo@gmail.com

José-Luis Gómez-Barroso, Universidad Nacional de Educación a Distancia (UNED), Facultad de CC Económicas y Empresariales.

Paseo Senda del Rey, 11. 28040 Madrid. jlgomez@cee.uned.es

Sergio Ramos-Villaverde, Noon Venture. Pasifae 9, 1B. 28022 Madrid http://www.noonventure.com/ sergio.ramos@noonventure.com 